\title{
Re-assessing the rule of $\mathbf{5}$, two decades on
}

In 1997 Christopher Lipinski and colleagues looked at the physicochemical characteristics of approved drugs and clinical candidates at that time, and proposed that the 'rule of 5 ' could predict the likelihood that a given small molecule will be orally active. This guideline prioritized compounds that have molecular masses of less than 500 daltons, calculated logarithm of the octanol-water partition coefficient (clogP) of less than 5, 5 or fewer hydrogen bond donors, and 10 or fewer hydrogen bond acceptors - and red flagged compounds that have more than one parameter out of range. Novartis's Michael Shultz now argues that this drug-likeness rule of thumb does not stand the test of time.

Reporting in the Journal of Medicinal Chemistry, Shultz assessed the physicochemical properties of the 409 small molecules that the FDA has approved since 1997. Molecular mass in this cohort has increased considerably from the baseline, he found, and in both 2016 and 2017 the average molecular mass of FDA-approved drugs was greater than 500 daltons. An up-to-date molecular mass cut-off based on the properties of orally available small molecules approved in the past decade would now be more than 600 daltons, he found. The threshold for hydrogen bond acceptors has also increased substantially.

These data call into question the hypothesis that 'drug-like' properties exist, he concludes. "Repeating the [rule of 5] experiment today, with over twice as many oral drugs approved than were available in 1997, gives different 'rules' than those hypothesized two decades ago ... If our past future predictions have not been accurate with the [rule of 5] parameters, we must call into question our current future predictions."

With new modalities coming online - such as targeted protein degraders and constrained peptides - he urges drug developers to reconsider how they use the rule of 5. "We are in danger of repeating our past mistakes if we assume these new modalities are not 'drug-like' and cannot be oral drugs because they are not [rule of 5] compliant," writes Shultz.

Asher Mullard

\section{How much do phase III trials cost?}

Drug development has been estimated to cost anywhere from $\underline{U S \$ 43 \text { million on }}$ one controversial end of the spectrum to $\$ 2.9$ billion on the other when failures, post-approval studies and opportunity cost are factored in. Taking a different approach to understanding these numbers, researchers have now focused more narrowly on the price tag for pivotal trials alone. The median expense for a single phase III trial is $\$ 19$ million, they report in JAMA Internal Medicine, after assessing the details of 138 pivotal trials for 59 new drugs that the FDA approved from 2015 to 2016.

Trial costs - estimated on the basis of a cost calculator from the IQVIA contract research organization - vary dramatically, they found. A four-patient phase III trial to test Wellstat's uridine triacetate for the rare hereditary metabolic disorder orotic aciduria likely cost only around $\$ 2$ million. But Novartis's 8,442-patient non-inferiority trial of sacubitril-valsartan versus enalapril, looking at hospitalization and cardiovascular mortality outcomes, likely cost around $\$ 347$ million.

"Our study provides a different perspective to the widely held assumption that elaborate and expensive clinical trials are the main reason for the high costs of developing a new drug. These data suggest that high-cost trials occur but usually when drug effects are small or a known drug already provides clinical benefit. On the other hand, pivotal trials for novel drugs with substantial clinical benefits can be conducted at a lower cost," the authors conclude.

This analysis did not capture some of the trial costs that are borne more directly by sponsors, such as the cost to manufacture drugs or the salaries for sponsor employees who oversee trials. The study wasn't designed to assess the costs of failed trials, of earlier-stage trials or of discovery projects, and was not adjusted to include the opportunity cost of these investments.

Nevertheless, the estimate is in line with previous work from analysts at KMR Group who have assessed the clinical trial costs of more than 700 clinical trials that were run by 7 major pharmaceutical companies between
2010 and 2015. The median phase III trial in their data set cost $\$ 21.4$ million, they reported last year in Nature Reviews Drug Discovery. The median phase II trial cost $\$ 8.6$ million, and the median phase I trial cost $\$ 3.4$ million, they also reported.

Asher Mullard

\section{FDA approves first drug under new antibacterial and antifungal drug programme}

The FDA's approval of Insmed's inhaled formulation of amikacin for lung disease associated with Mycobacterium avium marked the agency's first use of the Limited Population Pathway for Antibacterial and Antifungal Drugs (LPAD) approval pathway.

Congress established the LPAD under the 21st Century Cures Act as a means of promoting the development and approval of antibacterial and antifungal drugs for serious or life-threatening infections in limited patient populations with unmet need. Earlier in the year, the agency issued a draft guidance on the LPAD, noting that products that are reviewed under this pathway can follow streamlined clinical development strategies that may involve smaller, shorter or fewer clinical trials.

In the case of Insmed's inhaled amikacin, the regulatory green light was granted on the basis of a 336-patient, open-label phase III trial. Patients with refractory M. avium-associated lung disease were randomized to inhaled amikacin plus a background regimen of antibiotics, or to background antibiotics alone. At 6 months, $29 \%$ of amikacin-treated patients had no evidence of mycobacteria in their sputum, compared with $9 \%$ of patients on background therapy alone.

Injected formulations of amikacin have been in commercial use since the 1970s for the treatment of multidrug-resistant Gram-negative bacteria, non-tubercular mycobacterial infections and other indications.

"We're seeing a lot of early interest among sponsors in using this new pathway, and it's our hope that it'll spur more development and approval of antibacterial drugs for treating serious or life-threatening infections in limited populations of patients with unmet medical needs," says FDA commissioner Scott Gottlieb.

Asher Mullard 